\title{
Use of the VITEK 2 system to identify and test the antifungal susceptibility of clinically relevant yeast species
}

\author{
MSC Melhem, A Bertoletti, HRL Lucca, RBO Silva ${ }^{\dagger}$, FA Meneghin, MW Szeszs \\ Instituto Adolfo Lutz, Secretaria da Saúde, Governo do Estado de São Paulo, São Paulo, SP, Brazil.
}

Submitted: November 21, 2011; Approved: September 10, 2012.

\begin{abstract}
Eleven quality control isolates (Candida albicans ATCC 64548, C. tropicalis ATCC 200956, C. glabrata ATCC 90030, C. lusitaniae ATCC 200951, C. parapsilosis ATCC 22019, C. krusei ATCC 6258, C. dubliniensis ATCC 6330, Saccharomyces cerevisiae ATCC 9763, Cryptococcus neoformans ATCC 90012, C. gattii FIOCRUZ-CPF 60, and Trichosporon mucoides ATCC 204094) and 32 bloodstream isolates, including C. albicans, C. tropicalis, C. parapsilosis, C. glabrata, C. krusei, C. guilliermondii, C. pelliculosa (Pichia anomala), C. haemulonii, C. lusitaniae, and C. kefyr were identified at the species level by the VITEK 2 system. A set of clinical isolates (32 total) were used as challenge strains to evaluate the ability of the VITEK 2 system to determine the antifungal susceptibility of yeasts compared with the CLSI and EUCAST BMD reference standards. The VITEK 2 system correctly identified $100 \%$ of the challenge strains. The identification of yeast species and the evaluation of their susceptibility profiles were performed in an automated manner by the VITEK 2 system after approximately $15 \mathrm{~h}$ of growth for most species of Candida. The VITEK 2 system ensures that each test is performed in a standardized manner and provides quantitative MIC results that are reproducible and accurate when compared with the BMD reference methods. This system was able to determine the MICs of amphotericin B, flucytosine, voriconazole, and fluconazole in $15 \mathrm{~h}$ or less for the most common clinically relevant Candida species. In addition, the VITEK 2 system could reliably identify resistance to flucytosine, voriconazole, and fluconazole and exhibits excellent quantitative and qualitative agreement with the CLSI or EUCAST broth microdilution reference methods.
\end{abstract}

Key words: antifungal agents, Candida, susceptibility testing methods, MIC, CLSI, EUCAST.

\section{Introduction}

The incidence of invasive nosocomial fungal infections caused by yeasts has been significantly increasing over the last two decades, resulting in high morbidity and mortality (Espinel-Ingroff et al., 2005). Although Candida albicans is the primary etiological agent of candidemia worldwide, non-C. albicans species are likely to overtake C. albicans in some hospital units, particularly those in Brazilian hospitals (Perkins et al., 2005). Among the nonC. albicans species, C. tropicalis, C. parapsilosis and $C$. glabrata are the most common causative agents of candidemia in Brazil (Meyer et al., 2009). The prognosis of candidemia depends on the immunological status of the host, on the virulence of the fungal species, on the resistance of the causative strain to antifungals and on the efficacy of antifungal therapy (Pereira et al., 2010). Candida glabrata is known to have acquired resistance to fluconazole and other azole drugs. Intrinsic resistance to older azoles has been well established for C. krusei, and primary resistance to amphotericin has been described in species, such as C. lusitaniae and C. haemulonii (RodriguezTudela et al., 2008). Concern about antifungal resistance, particularly with azole-class agents, amphotericin B and echinocandins, a new class of antifungal, necessitates the accurate in vitro susceptibility testing of medically relevant yeasts (Clinical and Laboratory Standards Institute, 2008; Diekema et al., 2009). 
The susceptibility patterns of Candida spp. are often unknown, and empiric therapy is frequently used to treat infections. Early species identification and rapid antifungal susceptibility testing (AFST) are needed in cases of critical infections. Standardized broth microdilution methods for AFST, published by the Clinical and Laboratory Standards Institute (CLSI) and the European Committee on Antimicrobial Susceptibility Testing (EUCAST), have served as the standard of reference for AFST (Rodriguez-Tudela et al., 2008; Verweij et al., 1999).

Although a number of AFST systems have been developed and are commercially available, their performance is variable (Buchaille et al., 1998; Cuenca-Estrella et al., 2005; Hata et al., 2007; Zaragoza et al., 2011). The VITEK 2 system (bioMérieux, Inc., Hazelwood, MO) is a fully automated system that permits both species identification and antifungal susceptibility testing. (Pfaller et al., 2007; Revankar et al., 1998). The aim of this study is to evaluate the commercial VITEK 2 system for species identification and antifungal susceptibility testing of Brazilian bloodstream isolates. The minimum inhibition concentration (MIC) results obtained using the VITEK 2 system were compared with those obtained using the CLSI and EUCAST reference procedures. For the CLSI method, results were calculated at both 24 and $48 \mathrm{~h}$ of incubation. Agreement between the antifungal drug susceptibility results obtained using the VITEK 2 system and those obtained using each BMD method ( $\pm 2 \log _{2}$ dilutions) was assessed by combining the data obtained from both the quality control and from the challenge clinical isolates in all tests (Cuenca-Estrella et al., 2010).

\section{Materials and Methods}

\section{Study design}

The purpose of this study was to evaluate the utility of the commercially available VITEK 2 system for the identification of medical yeasts at the species level. This study was also designed to compare the MIC results obtained by the VITEK 2 method for fluconazole, voriconazole, amphotericin B, and flucytosine to those obtained by the CLSI and EUCAST BMD methods (Clinical and Laboratory Standards Institute, 2008; Verweij, 1999). Furthermore, this study uses the recent recommendations published by EUCAST for the testing of non-fermentative yeasts, e.g., Cryptococcus. and Trichosporon species. The intralaboratory reproducibility of the identification tests and of the antifungal susceptibility tests was determined by testing all isolates in triplicate on three separate days. The MIC results obtained using the VITEK 2 system following 10 to $30 \mathrm{~h}$ of incubation (depending on the growth rate of the organism) were compared to those obtained using the CLSI reference BMD method after 24 and $48 \mathrm{~h}$ of incubation and using the EUCAST BMD method after $24 \mathrm{~h}$ of incubation (Cuenca-Estrella et al., 2010).

\section{Test organisms}

The test organisms evaluated in this study included 11 American Type Culture Collection (ATCC) strains that have been established as QC strains for species identification tests: C. albicans ATCC 64548, C. tropicalis ATCC 200956, C. glabrata ATCC 90030, C. lusitaniae ATCC 200951, C. parapsilosis ATCC 22019, C. krusei ATCC 6258, C. dubliniensis ATCC 6330, Saccharomyces cerevisiae ATCC 9763, C. neoformans ATCC 90012, C. gattii FIOCRUZ-CPF 60, and Trichosporon mucoides ATCC 204094. The challenge strains used for the species identification and antifungal susceptibility tests were bloodstream clinical isolates $(n=32)$ of $C$. albicans $(\mathrm{n}=5)$, C. tropicalis $(\mathrm{n}=5), C$. parapsilosis $(\mathrm{n}=5)$, C. glabrata $(\mathrm{n}=5), C$. krusei $(\mathrm{n}=5), C$. guilliermondii $(\mathrm{n}=3), C$. pelliculosa (Pichia anomala) $(\mathrm{n}=1), C$. haemulonii $(\mathrm{n}=1)$, C. lusitaniae $(\mathrm{n}=1)$, and C. $k$ efyr $(\mathrm{n}=1)$. These strains are all recent clinical isolates and were selected to represent clinically prevalent fungal species, including fluconazoleresistant strains and amphotericin B-resistant species. These isolates were selected to provide on-scale azole MICs ranging from 0.12 - > $64 \mathrm{mg} / \mathrm{L}$, voriconazole MICs from 0.06 to $4 \mathrm{mg} / \mathrm{L}$, flucytosine $\mathrm{MICs}$ from 0.015-16 mg/L, and amphotericin B MICs from 0.015-4 $\mathrm{mg} / \mathrm{L}$. All isolates were previously identified by the API 20C AUX test (bioMérieux) or by conventional methods (Matta et al., 2007).

\section{Identification tests}

Prior to testing, a suspension of each isolate was inoculated at least twice onto Can 2 chromogenic agar plates (bioMérieux, France) and onto Sabouraud dextrose agar slants to ensure the purity and the viability of the cultures. The inoculum suspensions for the VITEK 2 were prepared in sterile saline at a turbidity equal to a $2.0 \mathrm{McFarland}$ standard, as measured using a DensiChek instrument (bioMérieux). The individual test cards were automatically filled with the prepared culture suspension, sealed, and incubated by the VITEK 2 instrument. The cards were incubated at $35.5^{\circ} \mathrm{C}$ for $18 \mathrm{~h}$, and optical density readings were taken automatically every $15 \mathrm{~min}$. The final profile results were compared with the database, and the identification of the unknown organism was obtained.

A final identification of "excellent," "very good," "good," "acceptable," or "low- discrimination" was considered to be correct. The clinical isolates were previously identified at the species level by the API 20C AUX system (bioMérieux), which utilizes 20-cupule plastic strips containing dehydrated carbohydrate substrates. Conventional plate assimilation tests (auxanograms) for carbon use were also performed on these isolates using the following 27 compounds: glucose, L-sorbose, galactose, D-ribose, Dxylose, L-arabinose, D-arabinose, L-rhamnose, salicin, melibiose, sucrose, alpha-methil-D-glucoside, inositol, maltose, trehalose, cellobiose, lactose, raffinose, D-glu- 
citol, ribitol, erythritol, glycerol, melezitose, D-mannitol, galactitol, D-L lactic acid, and D-L-succinic acid. Plate assimilation tests for nitrogen use were performed with peptone and potassium nitrate. In addition, yeast isolates were evaluated for fermentation using Durham tubes and for urease activity. Filamentous growth and chlamydospore production were assessed on corn meal agar (Matta et al., 2007).

Both the conventional tests and the tests performed using the API 20 C AUXA were considered to be reference standards for this study, as both approaches were able to correctly identify the clinical isolates at the species level. The two approaches were evaluated independently, and personnel were blinded to the identity of the isolates.

\section{Antifungal agents}

The VITEK 2 cards containing serial twofold dilutions of amphotericin B, fluconazole, flucytosine, and voriconazole were provided by the manufacturer. For this study, microdilution plates containing serial twofold dilutions of each drug were prepared according to the CLSI or EUCAST BMD recommendations. The following antifungal compounds were included in the BMD assay: amphotericin B (0.03-16 $\mu \mathrm{g} / \mathrm{mL}$, Sigma-Aldrich), flucytosine (0.12-64 $\mu \mathrm{g} / \mathrm{mL}$, Sigma-Aldrich), fluconazole $(0.12-64 \mu \mathrm{g} / \mathrm{mL}$, Pfizer S.A., NY), and voriconazole (0.015-8 $\mu \mathrm{g} / \mathrm{mL}$, Pfizer S.A., NY). A stock solution of each antifungal agent was prepared in two-milliliter aliquots in either dimethyl-sulfoxide (amphotericin B and voriconazole) or in distilled water (fluconazole and flucytosine). The media used for the final drug dilutions was RPMI 1640 with potassium bicarbonate and without L-glutamine, buffered to $\mathrm{pH} 7$ using $165 \mathrm{mM}$ MOPS buffer (SigmaAldrich). The media were prepared as $2 \mathrm{x}$ stocks, and $100 \mu \mathrm{L}$ was added to each well of the microdilution plates. The plates were sealed and were stored at $-80^{\circ} \mathrm{C}$ until use.

\section{Antifungal susceptibility testing (AST)}

Yeast inocula were prepared in sterile distilled water from a 24-h (Candida spp.) or from a 48-h culture (Cryptococcus spp.) and were incubated on Sabouraud dextrose agar at $35^{\circ} \mathrm{C}$ or $30{ }^{\circ} \mathrm{C}(C$. spp.). The inocula for the VITEK 2 were prepared in sterile saline to a turbidity equal to a $2.0 \mathrm{McF}$ arland standard according to a bioMérieux DensiChek instrument. Each standardized inoculum suspension was placed into a VITEK 2 cassette along with a sterile polystyrene test tube and a yeast susceptibility test card. The cassettes were placed in the VITEK 2 instrument and the respective yeast suspensions were diluted appropriately, after which the cards were filled, incubated, and read automatically by the VITEK 2 . The time of incubation varied from 10 to $30 \mathrm{~h}$ based on the growth rate in the drug-free control well, and the results were expressed as MICs in micrograms per milliliter.
The inocula for the BMD reference assays were prepared by diluting a portion of the suspension that was prepared for the VITEK 2 assay to a turbidity equivalent to a $0.5 \mathrm{McF}$ arland standard. The turbidity of each inoculum was measured spectrophotometrically, and suspensions of $10^{6} \mathrm{cfu} / \mathrm{mL}$ were prepared. These suspensions were further diluted to yield a final concentration for each inoculum, as indicated by the CLSI document M27-A3 (1-5 x $10^{3}$ $\mathrm{cfu} / \mathrm{mL})$ or by the EUCAST $\left(1-5 \times 10^{5} \mathrm{cfu} / \mathrm{mL}\right)$ BMD method. The final inocula were inoculated in the wells of the BMD plates containing antifungals. The plates were incubated at $35^{\circ} \mathrm{C}$ (Candida spp.) or at $30^{\circ} \mathrm{C}(\mathrm{C}$. spp.) for up to $48 \mathrm{~h}$. The plates prepared by the EUCAST BMD method were read at $24 \mathrm{~h}$ of incubation, and those prepared by the CLSI BMD method were read at both 24 and $48 \mathrm{~h}$ of incubation. The optical density (OD) of each well was assessed spectrophotometrically (Titertek Multiskan, Sweden) at $492 \mathrm{~nm}$. MIC values for fluconazole, voriconazole, and flucytosine were defined as the lowest concentration of antifungal that resulted in a $50 \%$ reduction in the OD relative to the drug-free control. For amphotericin B, the MIC was defined as the concentration of antifungal that reduced growth by $100 \%$ (CLSI method) or by $90 \%$ (EUCAST method) compared with the control $(3,23,25)$. $C$. parapsilosis ATCC 22019 and C. krusei ATCC 6258 were included in all VITEK 2 and CLSI or EUCAST BMD assays to provide on-scale MIC results and to represent fluconazole-susceptible and fluconazole-resistant species, respectively. In this study, all MICs by strain were in their respective reference ranges.

\section{Analysis of results}

The reproducibility and accuracy of the results obtained using the VITEK 2 system compared to the reference techniques were calculated to determine a percentage of essential agreement (A) between the species identification and the MIC values. Discrepancies among MIC endpoints of more than $\pm 2 \log _{2}$ dilutions were used to calculate the essential agreement (Cuenca-Estrella et al., 2010; Pfaller et al., 2007).

\section{Results}

All eleven type strain isolates and all 32 bloodstream clinical isolates demonstrated sufficient growth at $15 \mathrm{~h}$ of incubation, and the species could be determined by the VITEK 2 system at different incubation periods. The VITEK 2 system met the desired reproducibility criteria (100\% correct) for the identification of all QC strains at the species level. Two of the 32 clinical isolates tested $(C$. guilliermondii and $C$. glabrata) yielded low-discrimination (but correct) results and required supplemental observation and testing to resolve their identities to a single taxon. Following additional tests, as recommended by the manufacturer, all 32 challenge strains were successfully identified 
by the VITEK 2 system. No isolates failed to grow in the VITEK 2 system in any of the replicates in any test.

All eleven type strain isolates and thirty one bloodstream clinical isolates were correctly identified at the species level by the VITEK 2 system. One Cryptococcus isolate that was recently reclassified by molecular methodologies as a new species - C. gattii - was identified as $C$. neoformans. The two Cryptococcus type strains and the Trichosporon mucoides type strain exhibited the characteristics of slow-growing organisms.

To assess the performance of the VITEK 2 system for the determination of MICs, we evaluated 32 clinical isolates. The VITEK 2 MIC results were produced after 14 to $27 \mathrm{~h}$ of incubation for all Candida spp. The mean timeto-result for the VITEK 2 system was $15 \mathrm{~h}$ for amphotericin $\mathrm{B}$ (with a range of 11 to $27.8 \mathrm{~h}$ ) and fluconazole (with a range of 9 to $24.2 \mathrm{~h}$ ), $13.1 \mathrm{~h}$ for flucytosine (with a range of 13.0 to $27 \mathrm{~h}$ ), and $12.4 \mathrm{~h}$ for voriconazole (with a range of 8.1 to $25.1 \mathrm{~h}$ ). All except 7 of the 32 clinical isolates ( 5 isolates of C. krusei, C. kefyr, and C. pelliculosa) demonstrated sufficient growth at $24 \mathrm{~h}$ of incubation for the CLSI BMD method, allowing the majority of the MICs to be determined at this time point.

Table 1 summarizes the in vitro susceptibilities of 32 bloodstream clinical isolates of Candida spp. to amphotericin B, 5-flucytosine, fluconazole and voriconazole, as determined by the VITEK 2 system and by the two reference BMD methods (CLSI and EUCAST). For the CLSI method, the 24-h results are presented for all but seven isolates, which did not exhibit sufficient growth at this point; for these isolates, the results from $48 \mathrm{~h}$ are presented.

Table 1 - Antifungal susceptibilities of 32 bloodstream clinical isolates of Candida spp. as determined by the VITEK 2 system and the two reference CLSI and EUCAST BMD methodologies.

\begin{tabular}{|c|c|c|c|c|c|}
\hline \multirow[t]{2}{*}{ Species } & \multicolumn{5}{|c|}{ MIC (mg/L) } \\
\hline & Antifungal agent & Test method & Range & MIC 50 & $\mathrm{~A}(\%)^{*}$ \\
\hline \multirow[t]{12}{*}{ C. albicans (5) } & fluconazole & VITEK 2 & $\leq 1$ & & \\
\hline & & CLSI & $0.12-0.25$ & 0.12 & 100 \\
\hline & & AFST-EUCAST & $0.25-0.5$ & 0.25 & 100 \\
\hline & voriconazole & VITEK 2 & $\leq 0.12$ & & \\
\hline & & CLSI & $0.015-0.25$ & 0.015 & 100 \\
\hline & & AFST-EUCAST & $0.015-0.5$ & 0.03 & 100 \\
\hline & flucytosine & VITEK 2 & $\leq 1$ & & \\
\hline & & CLSI & $0.12-0.25$ & 0.12 & 100 \\
\hline & & AFST-EUCAST & $0.25-0.5$ & 0.25 & 100 \\
\hline & amphotericin B & VITEK 2 & $\leq 0.25-1$ & 0.5 & \\
\hline & & CLSI & $0.12-0.5$ & 0.25 & 100 \\
\hline & & AFST-EUCAST & $0.08-0.25$ & 0.06 & 100 \\
\hline \multirow[t]{12}{*}{ C. tropicalis (5) } & fluconazole & VITEK 2 & $\leq 1$ & & \\
\hline & & CLSI & $0.12-0.5$ & 0.12 & 100 \\
\hline & & AFST-EUCAST & $0.25-1$ & 0.25 & 100 \\
\hline & voriconazole & VITEK 2 & $\leq 0.12$ & & \\
\hline & & CLSI & $0.015-0.6$ & 0.06 & 100 \\
\hline & & AFST-EUCAST & $0.03-0.12$ & 0.03 & 100 \\
\hline & flucytosine & VITEK 2 & $\leq 1$ & & \\
\hline & & CLSI & $0.12-2$ & 0.12 & 100 \\
\hline & & AFST-EUCAST & $0.25-4$ & 0.25 & 100 \\
\hline & amphotericin B & VITEK 2 & $\leq 1$ & & \\
\hline & & CLSI & $0.12-0.5$ & 0.25 & 100 \\
\hline & & AFST-EUCAST & $0.25-1$ & 0.12 & 100 \\
\hline \multirow[t]{6}{*}{ C. glabrata (5) } & fluconazole & VITEK 2 & $2-\geq 64$ & 4 & \\
\hline & & CLSI & $1-\geq 64$ & 1 & 50 \\
\hline & & AFST-EUCAST & $1-\geq 64$ & 1 & 50 \\
\hline & voriconazole & VITEK 2 & $\leq 0.12-1$ & 0.12 & \\
\hline & & CLSI & $0.06-4$ & 0.12 & 100 \\
\hline & & AFST-EUCAST & $0.12-4$ & 0.12 & 100 \\
\hline
\end{tabular}


Table 1 (cont.)

\begin{tabular}{|c|c|c|c|c|c|}
\hline \multirow[t]{2}{*}{ Species } & \multicolumn{5}{|c|}{$\mathrm{MIC}(\mathrm{mg} / \mathrm{L})$} \\
\hline & Antifungal agent & Test method & Range & MIC 50 & $\mathrm{~A}(\%)^{*}$ \\
\hline & flucytosine & VITEK 2 & $\leq 1$ & & \\
\hline & & CLSI & $0.12-0.5$ & 0.12 & 100 \\
\hline & & AFST-EUCAST & $0.25-0.5$ & 0.25 & 100 \\
\hline & amphotericin B & VITEK 2 & $0.5-1$ & 0.5 & \\
\hline & & CLSI & $0.5-1$ & 0.5 & 100 \\
\hline & & AFST-EUCAST & $0.12-0.5$ & 0.25 & 100 \\
\hline \multirow[t]{12}{*}{ C. krusei (5) } & fluconazole & VITEK 2 & $16-32$ & 16 & \\
\hline & & CLSI & $16-32$ & 32 & 100 \\
\hline & & AFST-EUCAST & $16-32$ & 32 & 100 \\
\hline & voriconazole & VITEK 2 & $\leq 0.12-0.25$ & 0.25 & \\
\hline & & CLSI & $0.12-0.5$ & 0.5 & 100 \\
\hline & & AFST-EUCAST & $0.015-0.5$ & 0.25 & 100 \\
\hline & flucytosine & VITEK 2 & $8.0-16$ & 16 & \\
\hline & & CLSI & $4.0-16$ & 8 & 100 \\
\hline & & AFST-EUCAST & 8 & 8 & 100 \\
\hline & amphotericin B & VITEK 2 & $0.5-4$ & 0.5 & \\
\hline & & CLSI & 1 & 1 & 100 \\
\hline & & AFST-EUCAST & $0.5-2$ & 0.5 & 100 \\
\hline \multirow[t]{12}{*}{ C. parapsilosis (5) } & fluconazole & VITEK 2 & $\leq 1-4$ & $\leq 1$ & \\
\hline & & CLSI & $0.12-0.5$ & 0.12 & 80 \\
\hline & & AFST-EUCAST & $0.12-1$ & 0.5 & 100 \\
\hline & voriconazole & VITEK 2 & $\leq 0.12-0.25$ & $\leq 0.12$ & \\
\hline & & CLSI & $0.12-0.5$ & 0.15 & 100 \\
\hline & & AFST-EUCAST & $0.015-0.5$ & 0.06 & 100 \\
\hline & flucytosine & VITEK 2 & $\leq 1$ & $\leq 1$ & \\
\hline & & CLSI & 0.12 & 0.12 & 100 \\
\hline & & AFST-EUCAST & 0.25 & 0.25 & 100 \\
\hline & amphotericin B & VITEK 2 & $\leq 0.25-0.5$ & $\leq 0.25$ & \\
\hline & & CLSI & $0.12-0.5$ & 0.25 & 100 \\
\hline & & AFST-EUCAST & $0.06-0.5$ & 0.12 & 100 \\
\hline \multirow[t]{12}{*}{ C. lusitaniae (1) } & fluconazole & VITEK 2 & $\leq 1$ & & \\
\hline & & CLSI & 0.12 & & 100 \\
\hline & & AFST-EUCAST & 0.25 & & 100 \\
\hline & voriconazole & VITEK 2 & $\leq 0.12$ & & \\
\hline & & CLSI & 0.015 & & 100 \\
\hline & & AFST-EUCAST & 0.03 & & 100 \\
\hline & flucytosine & VITEK 2 & $\leq 1$ & & \\
\hline & & CLSI & 0.12 & & 100 \\
\hline & & AFST-EUCAST & 0.25 & & 100 \\
\hline & amphotericin B & VITEK 2 & $\geq 16$ & & 0 \\
\hline & & CLSI & 2 & & 0 \\
\hline & & AFST-EUCAST & 2 & & \\
\hline \multirow[t]{5}{*}{ C. dubliniensis (1) } & fluconazole & VITEK 2 & $\leq 1$ & & \\
\hline & & CLSI & 0.25 & & 100 \\
\hline & & AFST-EUCAST & 0.12 & & 100 \\
\hline & voriconazole & VITEK 2 & $\leq 0.12$ & & \\
\hline & & CLSI & 0.015 & & 100 \\
\hline
\end{tabular}


Table 1 (cont.)

\begin{tabular}{|c|c|c|c|c|c|}
\hline \multirow[t]{2}{*}{ Species } & \multicolumn{5}{|c|}{$\mathrm{MIC}(\mathrm{mg} / \mathrm{L})$} \\
\hline & Antifungal agent & Test method & Range & MIC 50 & $\mathrm{~A}(\%)^{*}$ \\
\hline & & AFST-EUCAST & 0.03 & & 100 \\
\hline & flucytosine & VITEK 2 & $\leq 1$ & & \\
\hline & & CLSI & 0.12 & & 100 \\
\hline & & AFST-EUCAST & 0.25 & & 100 \\
\hline & amphotericin B & VITEK 2 & $\leq 0.25$ & & \\
\hline & & CLSI & 0.25 & & 100 \\
\hline & & AFST-EUCAST & 0.12 & & 100 \\
\hline \multirow[t]{12}{*}{ C. haemunolii $(\mathrm{n}=1)$} & fluconazole & VITEK 2 & $\geq 64$ & & \\
\hline & & CLSI & 0.12 & & 0 \\
\hline & & AFST-EUCAST & 0.12 & & 0 \\
\hline & voriconazole & VITEK 2 & 1 & & \\
\hline & & CLSI & 0.015 & & 0 \\
\hline & & AFST-EUCAST & 0.03 & & 0 \\
\hline & flucytosine & VITEK 2 & $\leq 1$ & & \\
\hline & & CLSI & 0.25 & & 100 \\
\hline & & AFST-EUCAST & 0.5 & & 100 \\
\hline & amphotericin B & VITEK 2 & $\geq 16$ & & \\
\hline & & CLSI & 8 & & 100 \\
\hline & & AFST-EUCAST & 4 & & 100 \\
\hline \multirow[t]{12}{*}{ C. kefyr $(\mathrm{n}=1)$} & fluconazole & VITEK 2 & 2 & & \\
\hline & & CLSI & 0.5 & & 100 \\
\hline & & AFST-EUCAST & 0.5 & & 100 \\
\hline & voriconazole & VITEK 2 & $\leq 0.12$ & & \\
\hline & & CLSI & 0.03 & & 100 \\
\hline & & AFST-EUCAST & 0.03 & & 100 \\
\hline & flucytosine & VITEK 2 & $\leq 1$ & & \\
\hline & & CLSI & 0.12 & & 100 \\
\hline & & AFST-EUCAST & 0.12 & & 100 \\
\hline & amphotericin B & VITEK 2 & 2 & & \\
\hline & & CLSI & 0.25 & & 0 \\
\hline & & AFST-EUCAST & 0.25 & & 0 \\
\hline \multirow[t]{12}{*}{ C. pelliculosa $(\mathrm{n}=1)$} & fluconazole & VITEK 2 & 2 & & \\
\hline & & CLSI & 2 & & 100 \\
\hline & & AFST-EUCAST & 2 & & 100 \\
\hline & voriconazole & VITEK 2 & $\leq 0.12$ & & \\
\hline & & CLSI & 0.25 & & 100 \\
\hline & & AFST-EUCAST & 0.25 & & 100 \\
\hline & flucytosine & VITEK 2 & $\leq 1$ & & \\
\hline & & CLSI & 0.12 & & 100 \\
\hline & & AFST-EUCAST & 0.25 & & 100 \\
\hline & amphotericin B & VITEK 2 & $\leq 0.25$ & & \\
\hline & & CLSI & 0.12 & & 100 \\
\hline & & AFST-EUCAST & 0.12 & & 100 \\
\hline
\end{tabular}

*A, agreement ( \pm 2 Log 2 dilutions) between VITEK and broth microdilution method MICs. 


\section{Discussion}

All eleven quality control isolates were successfully identified using the VITEK 2 system. Quality control tests to assess the accuracy and reproducibility of this system yielded the expected results, covering the range of identifiable species according to the manufacturer's claims. Using clinical samples, the overall agreement between the VITEK 2 system and the reference methods (i.e., the conventional methods and the API $20 \mathrm{C}$ AUX) upon initial testing was $93.6 \%(29 / 31)$. In our study, two (6.5\%) low-discrimination identification results were obtained for $C$. guilliermondii and C. glabrata. Additional tests revealed that the identification of these two clinical isolates was correct, thus yielding the correct identification for all the clinical isolates tested.

When the results of the reference and challenge tests were combined, the VITEK 2 system correctly identified a total of 42 isolates (11 reference isolates and 31 challenge isolates) at the species level. Hata et al. (2007) discovered an error in identification using the VITEK 2 system for one clinical isolate of $C$. glabrata. However, we did not observe any misidentification or low-discrimination results for $C$. glabrata or even for C. krusei, C. parapsilosis, and C. tropicalis (Hazen and Howell, 2003). In fact, the VITEK 2 system performed satisfactorily in the identification of non-Candida yeast species; isolates of Saccharomyces cerevisiae and Trichosporon mucoides were accurately identified by this system. Notably, Trichosporon species are somewhat refractory to the preparation of a uniform suspension in saline, leading to inaccurate results. A careful technique is required to achieve a homogeneous McFarland 2.0 turbidity, increasing the reproducibility of the results regardless the method employed.

The fact that $C$. gattii was not identified by the VITEK 2 system was expected, as this species is not present in the system database. The identification of this new species (formerly, C. neoformans var. gattii) requires additional molecular approaches (Pappas et al., 2004). Therefore, the identification of C. gattii, as C. neoformans is acceptable for this phenotypic approach; technical laboratory staff should be aware of this discrepancy and should submit clinical isolates to the appropriate reference center for genetic analysis as required. In this study, the identification of $C$. at the genus level using the VITEK 2 system was considered useful, as $C$. spp. cause serious, life-threatening meningeal cryptococcal infections. In vitro resistance to antifungals exhibited by members of this medically relevant genus is a concern among physicians (Pfaller et al., 2007). Although we could not determine MIC values for $C$. neoformans or $C$. gattii isolates due to insufficient growth at $30 \mathrm{~h}$, other authors have successfully employed this system to assess the susceptibility profile of $C$. neoformans strains (Cuenca-Estrella et al., 2010). From a clinical laboratory perspective, extensive work should be performed to determine the MIC profile for $C$. neoformans isolates such that these strains can be included in the VITEK 2 system database, which will improve its clinical utility.

In addition, we confirmed a clinically important advantage of the VITEK 2 system over conventional identification methods and over the API 20C AUXA system: species were identified within $18 \mathrm{~h}$ compared to $48-72 \mathrm{~h}$ for the other methods (Hazen et al., 2003).

The fluconazole MIC results obtained using the VITEK 2 system were highly reproducible, as determined by testing all 32 clinical isolates in triplicate on three different days (data not shown). The intralaboratory reproducibility of these results was $100 \%$ for all organisms tested, demonstrating the high level of standardization achieved using this automated microbiology system, which eliminates the subjectivity that could affect other approaches lacking this automation. The use of a spectrophotometer to provide objective readings of MICs at early time points was thoroughly established by the EUCAST committee and can also be employed for the CLSI BMD method (Clinical and Laboratory Standards Institute, 2008; Pfaller et al., 2007; Verweij et al., 1999).

Others have reported that the VITEK 2 system was able to determine fluconazole MIC endpoints after 9.1 to $27.1 \mathrm{~h}$ of incubation (mean, 12 to $14 \mathrm{~h}$ ). In this study, we confirmed this finding and observed a 15 -h median MIC endpoint for fluconazole. The determination of MIC values for C. neoformans, C. gattii and Trichosporon spp. by the VITEK 2 system required an incubation period greater than $30 \mathrm{~h}$, in agreement with a previous report (Cuenca-Estrella et al., 2010). It noteworthy that the VITEK 2 system was able to determine the MIC endpoints earlier than the BMD method, especially for $C$. tropicalis isolates, which required $18 \mathrm{~h}$ of incubation, as previously noted (Pfaller et al., 2007).

In this study, we confirm that the VITEK 2 system is able to reliably identify the high flucytosine, voriconazole, and fluconazole MICs that are characteristic of resistant fungal isolates. Recently, Pfaller et al. (2007) have shown that fluconazole may serve as a surrogate marker for voriconazole susceptibility in Candida spp. Elevated fluconazole MICs ([\#GTEQ\#] $64 \mathrm{mg} / \mathrm{L}$ ) predict the resistance of Candida spp. to voriconazole with an absolute categorical agreement of $97 \%, 0.1 \%$ very major errors, and $1.4 \%$ major errors (Posteraro et al., 2009). Therefore, testing for fluconazole susceptibility in a hospital setting using the VITEK 2 system can function as a surrogate test for voriconazole resistance.

The antifungal resistance results for the 32 clinical isolates are presented in Table 1. Fluconazole MICs for 25 clinical isolates at $24 \mathrm{~h}$, as determined by the CLSI BMD method were compiled for the agreement analysis, which was performed to reduce the likelihood of falsely elevated fluconazole MICs due to trailing growth (Clinical and Laboratory Standards Institute, 2008). Furthermore, previous work has shown that the fluconazole MIC value at $24 \mathrm{~h}$ cor- 
related better with the clinical outcome and with sterol quantification than the MIC value at $48 \mathrm{~h}(1,20)$. The MICs obtained using the VITEK 2 system were somewhat higher than those observed for the two reference methods. This finding may be because the range of antifungal concentrations used in this system does not match exactly the range used in the other techniques. In the case of fluconazole, for example, the reference method concentration range was 0.12 to $64 \mathrm{mg} / \mathrm{L}$, while the VITEK 2 concentration range was 1 to $64 \mathrm{mg} / \mathrm{L}$ ).

The overall agreement between the results obtained using the VITEK 2 system, and those obtained using the CLSI and EUCAST BMD reference methods were high (88.5\%). In recent studies, the VITEK 2 system has demonstrated a high level of reproducibility and an excellent categorical agreement with the CLSI microdilution reference procedure for fluconazole (at $95 \%$ ) and, more recently, for amphotericin B, flucytosine, and voriconazole (CuencaEstrella et al., 2010; Pfaller et al., 2007; Revankar et al., 1998).

Table 1 shows the agreement rates between procedures by antifungal agent. The rates of agreement were high when the results were analyzed per species. Regarding the individual species of Candida, the A between the VITEK 2 results and either of the BMD MICs was $100 \%$ for $C$. albicans, C. tropicalis, C. dubliniensis, C. pelliculosa (Pichia anomala), and C. guilliermondii. The A value is maximal for nearly all clinically relevant species except $C$. parapsilosis and C. glabrata, for which the A values were 97.5 and $87.5 \%$, respectively, between the results obtained using the VITEK 2 system and those obtained by the reference methods for all four antifungal drugs. For C. glabrata, the relatively poor agreement was due to the significantly (four-fold) higher fluconazole MICs for two isolates, as measured by the VITEK 2 system, compared to those obtained by both the CLSI and the EUCAST BMD methods. The agreement calculated for the fluconazole MIC in this study was lower (A, 50\%) than previously described (A, $89.2 \%$ ) by Pfaller et al. (2007). Given that C. glabrata is a species with known fluconazole resistance, these discrepancies should not pose a problem clinically.

Similar discrepancies were observed between the VITEK 2 system and the CLSI BMD method for one $C$. parapsilosis isolate. Notably, all three isolates (two $C$. glabrata and one C. parapsilosis isolate) exhibited trailing phenotypes, which may have a significant influence on the MIC determination by the VITEK 2 system for these two species. In this study, the trailing phenomenon, a wellknown technical artifact observed with fungistatic drugs, occurred in all C. tropicalis isolates and in the majority of C. glabrata and C. albicans isolates, regardless of the BMD method used. Trailing is a recognized factor that contributes to false positives for antifungal resistance, as this phenomenon results in residual fungal growth above the MIC endpoint. For this reason, MIC readings are typically mea- sured at $50 \%$ growth inhibition compared with the positive control. We observed that the VITEK 2 system, despite measuring fungal growth spectrophotometrically, yielded correct results even for the trailing phenotype frequently observed in C. tropicalis isolates.

In addition, the amphotericin B MICs generated by the VITEK 2 were higher than those obtained by the BMD method for $C$. lusitaniae and $C$. kefyr, and for $C$. haemulonii, another uncommon fungal species, the fluconazole and voriconazole MICs generated by the VITEK 2 system were fivefold higher than those obtained using the BMD method. These discrepancies emphasize the impact of trailing on BMD results obtained with these uncommon species and suggest that future studies will be required to better evaluate these emergent bloodstream isolates (Meyer et al., 2009; Rodriguez-Tudela et al., 2008). Notably, although we analyzed a smaller number of $C$. glabrata isolates, we did not observe a discrepancy in the voriconazole MIC for this species, as previously reported (Pfaller et al., 2007).

The only false-positive resistance results for the antifungal agent amphotericin B were observed with $C$. lusitaniae and C. kefyr. C. lusitaniae is a rare, emergent species of clinical concern due to its potential to develop resistance in vitro to amphotericin B. The high MIC value for C. lusitaniae, as determined by the VITEK 2 system, was not confirmed by the CLSI or EUCAST BMD methods, suggesting that a greater number of $C$. lusitaniae isolates will need to be evaluated to confirm our findings. Moreover, the artificially elevated fluconazole and voriconazole MIC values for this species were obtained using the VITEK 2 system, emphasizing the need to study this uncommon species in greater detail to confirm our observations and to contribute to a standardized test.

Interestingly, using the VITEK 2 system we observed a high amphotericin B MIC for a single clinical isolate of $C$. haemulonii that was also observed using the reference methods, confirming the resistance of this species to amphotericin B.

In this study of clinical bloodstream fungal isolates from Brazil, we confirm that the VITEK 2 system used to identify fungal species and to determine antifungal susceptibility is in excellent agreement with the reference BMD methods. The ability of the VITEK 2 system to provide quantitative MIC results is reproducible and accurate, identifying high MIC values that indicate resistance to flucytosine, voriconazole, and fluconazole. The MICs of fluconazole, voriconazole, amphotericin $\mathrm{B}$, and flucytosine could be determined rapidly $(\sim 15 \mathrm{~h})$ for most species of Candida using this system. The monitoring of the development of antifungal resistance during therapy should be assessed by AFST on a routine basis. The availability of rapid antifungal susceptibility data may play a major role in optimizing the therapy of invasive candidal infections. Furthermore, surveillance of antifungal susceptibility profiles pro- 
vides a useful tool for hospitals to validate empiric treatment regimens.

\section{Acknowledgments}

We thank Dra. Marcia Lazera from FIOCRUZ-RJ for sending the Cryptococcus gattii FIOCRUZ-CPF60 isolate. We acknowledge the excellent technical assistance of Andres Avelino Baez, Maria Angela de Souza, Catia R. Costa, I. T.J. Zanella, Jose Carlos Silva, and Olga M.Diniz of Instituto Adolfo Lutz laboratories.

\section{References}

Arthington-Skaggs BA, Lee-Yong W, Ciblak MA, Frade JP, Brandt ME, Hajjeh RA, Harrison LE, Sofair AN, Warnock DW (2002) Comparison of visual and spectrophotometric methods of broth microdilution MIC endpoint determination and evaluation of sterol quantitation method for in vitro susceptibility testing of fluconazole and itraconazole against trailing and non-trailing Candida isolates. Antimicrob Agents Chemother 46:2477-2481.

Buchaille L, Freydiere AM, Guinet R, Gille Y (1998) Evaluation of six commercial systems for identification of medically important yeasts. Eur J Clin Microbiol Infect Dis 17:479488.

Clinical and Laboratory Standards Institute (2008) Reference method for broth dilution antifungal susceptibility testing of yeast. Approved standard M27-A3, 3rd ed. Clinical and Laboratory Standards Institute, Wayne, P.

Cuenca-Estrella M, Gomez-Lopez A, Mellado E, RodriguezTudela JL (2005) Correlation between the procedure for antifungal susceptibility testing for Candida spp. of the European Committee on Antibiotic Susceptibility Testing (EUCAST) and four commercial techniques. Clin Microbiol Infect 11:486-492.

Cuenca-Estrella M, Gomez-Lopez A, Alastruey-Izquierdo A, Bernal-Martinez L, Cuesta I, Buitrago MJ, RodriguezTudela JL (2010) Comparison of the Vitek 2 Antifungal Susceptibility System with the Clinical and Laboratory Standards Institute (CLSI) and European Committee on Antimicrobial Susceptibility Testing (EUCAST) broth microdilution reference methods and with the sensititre yeastone and etest techniques for In Vitro detection of antifungal resistance in yeast isolates. J Clin Microbiol 48:1782-1786.

Diekema DJ, Messer SA, Boyken LB, Hollis RJ, Kroeger J, Tendolkar S, Pfaller MA (2009) In Vitro activity of seven systemically active antifungal agents against a large global collection of rare Candida species as determined by CLSI broth microdilution methods. J Clin Microbiol 47:31703177.

Espinel-Ingroff A, Barchiesi F, Cuenca-Estrella M, Pfaller MA, Rinaldi M, Rodriguez-Tudela JL, Verweij PE (2005) International and multicenter comparison of EUCAST and CLSI M27-A2 broth microdilution methods for testing susceptibilities of Candida spp. to fluconazole, itraconazole, posaconazole, and voriconazole. J Clin Microbiol 43:3884-3889.
Graf B, Adam T, Zill E, Gobel UB (2000) Evaluation of the VITEK 2 system for rapid identification of yeasts and yeast-like organisms. J Clin Microbiol 38:1782-1785.

Hata DJ, Hall L, Fothergill AW, Larone DH, Wengenack NL (2007) Multicenter Evaluation of the New VITEK 2 Advanced Colorimetric Yeast Identification Card. J Clin Microbiol 45:1087-1092.

Hazen KC, Howell SA (2003) Candida, Cryptococcus, and other yeasts of medical importance. In R. Murray, E. J. Baron, J. H. Jorgensen, M. A. Pfaller, and R. H. Yolken Manual of clinical microbiology 8:1693-1711, Washington, DC .

Matta DA, Almeida LP, Machado AM, Azevedo AC, Kusano EJU, Travassos SR, Colombo AL, NF (2007) Antifungal susceptibility of 1000 Candida bloodstream isolates to 5 antifungal drugs: results of a multicentes study conducted in São Paulo, Brazil, 1995-2003. Diagn Microbiol Infect Dis 57:399-404.

Meyer W, Aanensen DM, Boekhout T, Cogliati M, Diaz MR, Esposto MC, Fisher M, Gilgado Hagen F, Kaocharoen S, Litvintseva AP, Mitchell TG, Simwami SP, Trilles L, Viviani MA, Kwon-Chung KJ (2009) Consensus multi-locus sequence typing scheme for Cryptococcus neoformans and Cryptococcus gattii. Med Mycol 47:561-570.

Pappas PG, Rex JH, Sobel JD, Filler SG, Dismukes WE, Walsh TJ, Edwards JE (2004) Guidelines for treatment of candidiasis. Clin Infect Dis 38:161-189.

Pereira GH, Muller PR, Szeszs MW, Levin AS, Melhem MS (2010) Five-year evaluation of bloodstream yeast infections in a tertiary hospital: the predominance of non-C. albicans species. Med Mycol 48:839-842.

Perkins A, Gomez-Lopez A, Mellado E, Rodriguez-Tudela JL, Cuenca-Estrella M (2005) Rates of antifungal resistance among Spanish clinical isolates of Cryptococcus neoformans var. neoformans. J Antimicrob Chemother 56:1144-1147.

Pfaller MA, Diekema DJ, Procop GW, Rinaldi MG (2007) Multicenter comparison of the VITEK 2 yeast susceptibility test with the CLSI broth microdilution reference method for testing fluconazole against Candida spp. J Clin Microbiol 45:796-802.

Pfaller MA, Messer SA, Boyken L, Rice C, Tendolkar S, Hollis RJ, Diekema DJ (2007) The use of fluconazole as a "surrogate marker" to predict susceptibility and resistance to voriconazole among 13,338 clinical isolates of Candida spp. tested by Clinical and Laboratory Standards Institute recommended broth microdilution methods. J Clin Microbiol 45:70-75.

Posteraro B, Martucci R, La Sorda M, Fiori B, Sanglard D, De Carolis E, Florio AR, Fadda G, Sanguinetti M (2009) Reliability of the Vitek 2 yeast susceptibility test for detection of in vitro resistance to fluconazole and voriconazole in clinical isolates of Candida albicans and Candida glabrata. J Clin Microbiol 47:1927-1930.

Revankar SG, Kirkpatrick WR, McAtee RK, Fothergill AW, Redding SW, Rinaldi MG, Patterson TF (1998) Interpretation of trailing endpoints in antifungal susceptibility testing by the National Committee for Clinical Laboratory Standards method. J Clin Microbiol 36:153-156.

Rodero L, Cuenca-Estrella M, Cordoba S, Cahn P, Davel G, Kaufman S, Guelfand L, Rodriguez-Tudela JL (2002) Tran- 
sient fungemia caused by an amphotericin B-resistant isolate of Candida haemulonii. J Clin Microbiol 40:2266-2269.

Rodriguez-Tudela JL, Donnelly JP, Arendrup MC, Arikan S, Barchiesi F, Bille J, Chryssanthou E, Cuenca-Estrella M, Dannaoui E, Denning D, Fegeler W, Gaustad P, Lass-Florl C, Moore C, Richardson M, Schmalreck A, Velegraki A, Verweij P (2008) EUCAST technical note on the method for the determination of broth dilution minimum inhibitory concentrations of antifungal agents for conidia-forming moulds. Subcommittee on Antifungal Susceptibility Testing (AFST) of the ESCMID European Committee for Antimicrobial Susceptibility Testing (EUCAST). Clin Microbiol Infect 14:982-984.

Rodriguez-Tudela JL, Arendrup MC, Barchiesi F, Bille J, Chryssanthou E, Cuenca-Estrella M, Dannaoui E, Denning DW,
Donnelly JP, Dromer F, Fegeler W, Lass-Florl C, Moore C, Richardson M, Sandven P, Velegraki A, Verweij P (2008) EUCAST definitive document EDef 7.1: method for the determination of broth dilution MICs of antifungal agents for fermentative yeasts. Clin Microbiol Infect 14:398-405.

Verweij PE, Breuker IM, Rijs AJ, Meiss JF (1999) Comparative study of seven commercial yeast identification systems. J Clin Pathol 52:271-273.

Zaragoza O, Mesa-Arango AC, Gomez-Lopez A, Bernal-Martinez L, Rodriguez-Tudela JL, Cuenca-Estrella M (2011) A Process Analysis of Variables for Standardization of Antifungal Susceptibility Testing of Non-Fermentative Yeasts. Antimicrob Agents Chemother 55:1563-1570.

All the content of the journal, except where otherwise noted, is licensed under a Creative Commons License CC BY-NC. 